Contribution to the Seeheim Conference on Magnetism (SCM 2001), Seeheim,

Germany, Sept 9 -- 13, 2001, to appear in Physica Status Solidi A (2001)

\title{
Dipole coupling induced magnetic ordering in an ensemble of nanostructured islands
}

\author{
P. J. JENSEN*, ${ }^{*}$ and G. M. PASTOR \\ Laboratoire de Physique Quantique, Universite Paul Sabatier, UMR 5626 du CNRS \\ 118, route de Narbonne, F-31062 Toulouse, France \\ Subject classification: $75.10 . \mathrm{Nr}, 75.40 . \mathrm{Cx}, 75.75 .+\mathrm{a}$
}

${ }^{a}$ Tel./Fax: +33 (0)561556833 / 60 65; e-mail: jensen@irsamc.ups-tlse.fr

The magnetic ordering due to the long range dipole coupling in an ensemble of magnetic islands is investigated. If the islands are large enough and closely separated, the average dipole energy per island can explain the magnitude of the observed ordering temperature of such an ensemble (U. Bovensiepen et al., J. Magn. Magn. Mater. 192, L386 (1999)). The energetical degeneracy with respect to a continuous in-plane rotation of the magnetic moments in a periodic ensemble of islands is lifted in presence of an island size dispersion and an irregular island array. Many different (metastable) magnetic states are obtained, reminiscent of a spin-glass behavior. We obtain that the average magnetic binding energy per island due to the dipole coupling increases with increasing positional disorder. The island ensembles exhibit non-collinear magnetic structures, resulting in non-saturated ensemble magnetizations. The calculations are performed with a classical spin model for ensembles of islands in unit cells with periodic boundary conditions. The point dipole sums are augmented by an island areal correction.

Introduction The investigation of interacting magnetic nanoparticles is a very active field of current research both experimentally and theoretically [1] - [8]. Measurements on growing $\mathrm{Co} / \mathrm{Cu}(001)$ thin films exhibit a global ordering temperature of the order of $50-$ $100 \mathrm{~K}$, and a magnetic hysteresis and remanence also for coverages below the percolation threshold [1]. STM images taken in this coverage range yield an ensemble of double-layered Co islands with lateral size $\sim 5 \mathrm{~nm}$ and similar island separations, which should behave as a superparamagnetic ensemble. Blocking effects due to anisotropy barriers estimated by the Arrhenius-Néel-model [9] could be excluded in this temperature range. In this contribution we will examine whether the long range magnetic dipole coupling can be responsible for a long range magnetic ordering at these temperatures.

It is still under debate whether the magnetic dipole coupling can induce an equilibrium ordered state in an inhomogeneous two-dimensional (2D) or three-dimensional (3D) spin system [5] - [8]. Certainly, a simple ferro- or antiferromagnetic state will not evolve. Instead a spin-glass-type [10] (random magnet) is to be expected due to the nonuniform magnetic interactions, which are caused by the size- and shape dispersion as well as by the positional disorder of the island ensemble. In such a system many different metastable states exist, 
which are often characterized by extremely long relaxation times (magnetic viscosity).

The influence of the dipole coupling on the blocking effects have been studied extensively in the Arrhenius-Néel-framework for single particles, in particular for the investigation of dynamical processes. The results are satisfactory for weak interactions as compared to the anisotropy barriers of a single island [4] - [7]. For strong interactions, however, this model is no longer applicable. Here we will study the strongly interacting case, considering solely the dipole interaction. Note that the long range indirect exchange (RKKY-) interaction might also be responsible for the observed magnetic ordering in ensembles of magnetic islands.

Before describing the model let us first estimate whether the dipole coupling can account for the observed magnitude of the ordering temperature $T_{C}$, which must be of the order of the average magnetic binding energy per island. A pair of single spins has a dipole interaction strength of the order $E_{d i p} \sim \mu_{a t}^{2} / a_{o}^{3} \sim 1 \mathrm{~K}$, with $\mu_{a t} \sim 1 \mu_{B}$ the atomic magnetic moment, $\mu_{B}$ the Bohr magneton, and $a_{o} \sim 2.5 \AA$ the interatomic distance of $3 d$-transition metals. All $N$ spins of a magnetic island are aligned by the strong direct interatomic exchange coupling, and can be viewed as a single giant spin $M \sim N \cdot \mu_{a t}$ (Stoner-Wohlfarth particle [11]). The diameter of a flat (2D) islands is $L \sim \sqrt{N}$. In a densely packed island ensemble the island-island separation is $R \sim L$, resulting in an average magnetic dipole energy per island pair $E_{\text {dip }} \sim M^{2} / R^{3} \sim\left(\mu_{a t}^{2} / a_{o}^{3}\right) \sqrt{N}$. Thus, for a typical island size of about $N \sim 1000$ atoms the binding energy is $E_{\text {dip }} \sim 30 \mathrm{~K}$, yielding the correct order of magnitude. In the following we will determine $E_{\text {dip }}$ for a $2 \mathrm{D}$ island ensemble as functions of the size dispersion and positional disorder.

Theory For the calculation of the magnetic binding energy due to the dipole coupling in a 2D island array we consider a unit cell with $n$ non-overlapping, disk-shaped islands, and with periodic boundary conditions. Within the unit cell the islands can be placed using three different types of arrangements: (i) periodic square array; (ii) disturbed array, the island centers deviate from the sites of the periodic array using a Gaussian distribution with a standard deviation $\sigma_{r}$; (iii) random setup. The island size, which determines its lateral extension, can be either the same for all islands, of dispersed around a mean value $\bar{N}$ using also a Gaussian distribution with a standard deviation $\sigma_{N}$. For such an island ensemble we consider the following dipole-dipole Hamiltonian with classical magnetic moments $\mathbf{M}_{i}$ $\left(\left|\mathbf{M}_{i}\right|=N_{i} \cdot \mu_{a t}\right)$ :

$$
\mathcal{H}=\frac{1}{2} \sum_{\substack{i, j \\ i \neq j}} \frac{1}{r_{i j}^{5}}\left[\mathbf{M}_{i} \mathbf{M}_{j} r_{i j}^{2}-3\left(\mathbf{r}_{\mathbf{i j}} \mathbf{M}_{i}\right)\left(\mathbf{r}_{\mathbf{i j}} \mathbf{M}_{j}\right)\right]
$$

$r_{i j}=\left|\mathbf{r}_{i j}\right|=\left|\mathbf{r}_{i}-\mathbf{r}_{j}\right|$ being the distance between the centers of gravity of islands $i$ and $j$. The infinite range of the dipole interaction is taken into account by applying an Ewald type summation over all periodically arranged unit cells of the infinitely extended thin film. Since for a large coverage the islands are closely separated, we consider in addition to the usual point dipole sum the so-called areal correction (dipole-quadrupole interaction). This is of the order of $\left(A_{i}+A_{j}\right) / r_{i j}^{2}$, where $A_{i} \propto N_{i}$ is the area of island $i$ [12], and amounts up to $50 \%$ of the average dipole energy. For spherically shaped islands this correction vanishes since a 
sphere has no quadrupole moment [13]. We neglect here the lattice anisotropy. The shape anisotropy due to the dipole coupling within a single island vanishes for disk- or sphereshaped islands. For the atomic magnetic moments and the interatomic distances we choose values appropriate for Fe: $\mu_{a t}=2.2 \mu_{B}$ and $a_{o}=2.5 \AA$.

Starting from an arbitrary initial guess the total magnetic energy is minimized by varying the magnetic directions of each island. This magnetic relaxation is performed with the help of a conjugated gradient method. For simplicity, we restrict the island magnetizations to be always directed in-plane, $\mathbf{M}_{i}=M_{i}\left(\cos \phi_{i}, \sin \phi_{i}, 0\right)$, characterized by its in-plane (azimutal) angle $\phi_{i}$. To account for the many different energy minima in case of a nonuniform spatial island arrangement or in presence of an island size dispersion, the magnetic energy is averaged over many different initial guesses for the same island arrangement. In addition, we average $E_{d i p}$ over ten different realizations of the unit cell, using the same global variables (average sizes, standard deviations, etc.) characterizing the island ensemble. The energy reference is given by a random set of magnetic angles $\left\{\phi_{i}\right\}$, refering to a completely disordered system.

Results Before presenting the average magnetic dipole energy in particular for a disordered island array, let us recall shortly the resulting structure for a uniform system, i.e. the islands having all the same size and placed on a square mesh. Two different magnetic arrangements are possible: (i) a metastable parallel magnetization of the islands (ferromagnetic solution), and (ii) a columnar arrangement consisting of magnetized rows (or columns) of islands with alternating magnetic orientations. The latter is the ground state of a square array of spins or magnetic islands, having a vanishing remanent magnetization. Note that both solutions exhibit a continuous degeneracy with respect to an in-plane rotation. This property is one of the prerequisites for a vanishing global magnetic order of a $2 \mathrm{D}$ magnetic system at finite temperatures (Mermin-Wagner-theorem [14]). By introducing now a size- or positional disorder into the island ensemble, this degeneracy is immediately lifted. The system exhibits a (possibly very large) number of discrete states, which are separated by energy barriers and have approximately the same energy if no external magnetic fields or aligned anisotropy easy axes are present. The magnetic arrangement of the island ensemble is strongly noncollinear, the net magnetization is small or vanishes completely (demagnetizing effect due to flux closure), cf. Fig.1. We emphasize that these non-collinear arrangements do not correspond to a disordered system, since the local magnetizations are strongly correlated by the dipole interaction. The parallel and the columnar magnetic arrangements of the island ensemble are always unfavorable, and even do no longer refer to a stable state. Furthermore, due to the lifting of the energy degeneracy the above mentioned prerequisite for the MerminWagner-theorem is no longer fulfilled. Therefore, a dipole induced magnetic order might exist at finite temperatures also for an inhomogeneous 2D system ('order-by-disorder-effect' [15]).

In Fig. 2 we show the average magnetic dipole energy $E_{\text {dip }}$ per island as a function of the island positional disorder, characterized by the standard deviation $\sigma_{r}$. The (average) island size is chosen to be $N=100$, the average island-island distance is 1.5 times the (average) island diameter, resulting in a coverage of about $35 \%$. $E_{d i p}$ decreases with increasing positional disorder, reaching its minimal value (= maximal magnetic binding energy per island) for a random setup of the island ensemble. The increase of binding energy with 
respect to the uniform system amounts to roughly $50 \%$. As can be also seen from Fig.2, an island-size dispersion has no large effect on $E_{d i p}$.

The reason for the different effects of the two types of disorder on the magnetic binding energy is that $E_{d i p}$ is a bilinear function of the island sizes $N_{i}$, the size dispersion effect averages out for symmetrically distributed island sizes around the mean value $\bar{N}$. On the other hand, the dipole energy has a nonlinear dependence on the island-island distance: $E_{\text {dip }} \propto r_{i j}^{-3}$. Thus, with increasing positional disorder the decrease of $\left|E_{\text {dip }}\right|$ with an enlarged distance $r_{i j}$ between some island pairs is more than counterbalanced by a corresponding increase of $\left|E_{\text {dip }}\right|$ for smaller distances between other island pairs. This leads to an increase of the average magnetic binding energy of the island ensemble.

Conclusion We have calculated the magnetic binding energy due to the long range dipole coupling in a 2D ensemble of magnetic islands. The binding energy increases by ca. $50 \%$ for a random arrangement of islands with respect to a periodic array. An island size dispersion has less effect on the binding energy. Due to the nonuniform system a large number of different metastable states emerges with strongly non-collinear magnetic arrangements, as reminiscent of a spin-glass [10]. We expect that if the islands are large enough and closely separated, the dipole energy can explain the observed magnetic ordering of a growing thin film below its percolation threshold [1]. As a first approximation, the resulting ordering temperature can be determined from a mean-field approximation, and corresponds to the spin-glass temperature. For a comparison with experiments also magnetic anisotropies need to be taken into account, as well as overlapping magnetic islands [16]. Furthermore, one can calculate the ensemble magnetization and susceptibility as a function of an applied magnetic field.

Acknowledgement The authors acknowledge financial support from CNRS (France), and by the EU GROWTH project AMMARE under contract number G5RD-CT-2001-00478. 


\section{References}

[*] On leave from: Institut für Theoretische Physik, Freie Universität Berlin, Arnimallee 14, D-14195 Berlin, Germany.

[1] U. BOVENSIEPEN et al., J. Magn. Magn. Mater. 192, L386 (1999).

[2] S. SUN et al., Science 287, 1989 (2000).

[3] M. RESPAUD et al., Phys. Rev. B 57, 2925 (1998); M. GIERSIG and M. HILGERSDORFF, J. Phys. D 32, L111 (1999); V. RUSSIER et al., Phys. Rev. B 62, 3910 (2000).

[4] R. W. CHANTRELL et al., J. Magn. Magn. Mater. 157/158, 250 (1996); Phys. Rev. B 63, 024410 (2000); D. KECHRAKOS and K. N. TROHIDOU, Phys. Rev. B 58, 12169 (1998).

[5] J. O. ANDERSSON et al., Phys. Rev. B 56, 13983 (1997); T. JONSSON et al., Phys. Rev. B 57, 497 (1998); D. FIORANI et al., J. Magn. Magn. Mater. 196-197, 143 (1999).

[6] M. F. HANSEN and S. MØRUP, J. Magn. Magn. Mater. 184, 262 (1998); J. L. DORMANN, D. FIORANI, and E. TRONC, J. Magn. Magn. Mater. 202, 251 (1999).

[7] M. A. ZAŁUSKA-KOTUR, Phys. Rev. B 54, 1064 (1996).

[8] H. ZHANG and M. WIDOM, Phys. Rev. B 51, 8951 (1995).

[9] L. NÉEL, Ann. Geophys. 5, 99 (1949); W. F. BROWN, Jr., Phys. Rev. 130, 1677 (1963).

[10] K. BINDER and A. P. YOUNG, Rev. Mod. Phys. 58, 801 (1986).

[11] E. C. STONER and E. P. WOHLFARTH, Trans. Roy. Soc. A 240, 599 (1948).

[12] P. J. JENSEN, unpublished.

[13] Y. G. POGORELOV, private communication.

[14] N. M. MERMIN and H. WAGNER, Phys. Rev. Lett. 17, 1133 (1966).

[15] J. VILlain, Z. Phys. B 33, 31 (1979); C. L. HENLEY, Phys. Rev. Lett. 62, 2056 (1989); S. PRAKASH and C. L. HENLEY, Phys. Rev. B 42, 6574 (1990).

[16] P. POUlOPOUlOS et al., Phys. Rev. B 65 (2002). 


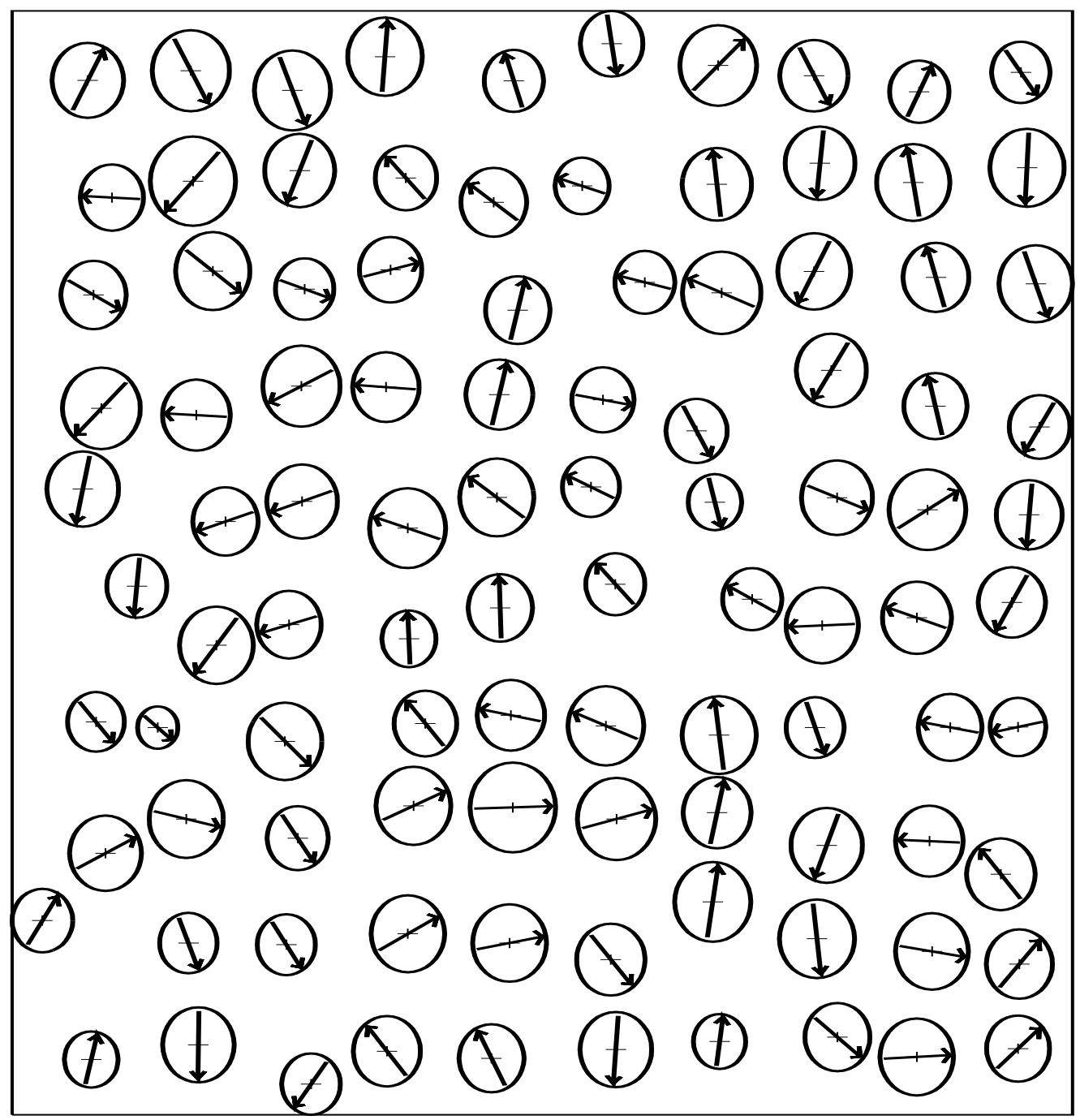

Figure 1: Visualization of a typical (meta-) stable magnetic arrangement in the unit cell as obtained from the calculations for the average dipole energy. The average island size is $\bar{N}=100$ spins, yielding an average island radius of about $10 a_{o}, a_{o}$ the interatomic distance. The size of the unit cell is chosen to be $\left(150 a_{o}\right) \times\left(\begin{array}{ll}150 & a_{o}\end{array}\right)$, containing 100 islands, and yielding a coverage $\Theta \sim 35 \%$. The spatial island arrangement corresponds to a disturbed array, the island centers deviate from the sites of a square array with a standard deviation $\sigma_{r}=10 \%$. The island size exhibits a dispersion of about $\sigma_{N}=20 \%$. The arrows depict the in-plane directions of the island magnetizations. Note the tendency to the formation of island chains and flux closure structures. 


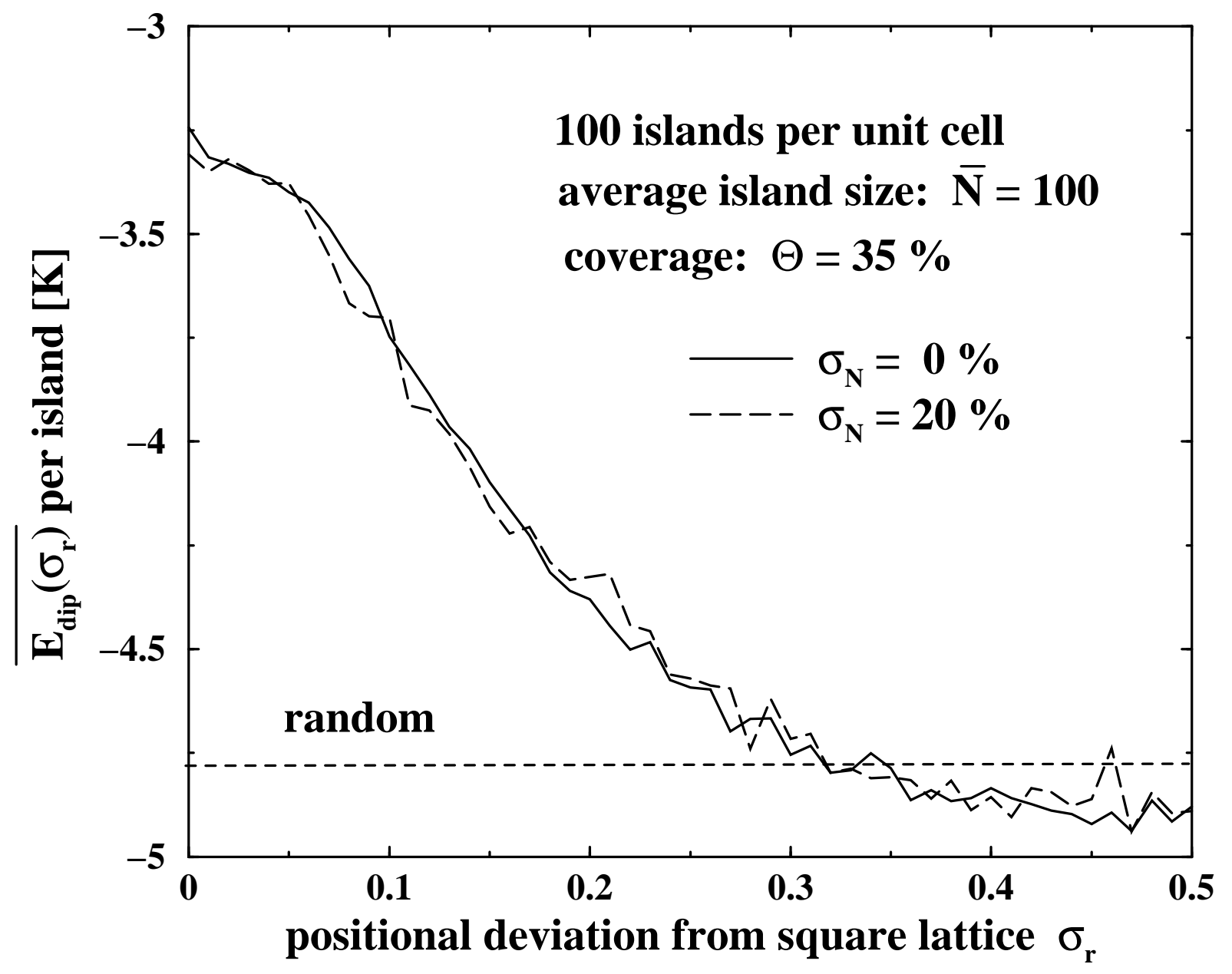

Figure 2: Average dipole energy $\overline{E_{d i p}\left(\sigma_{r}\right)}$ per island of a 2D ensemble of magnetic islands as a function of the positional disorder, which is measured by its standard deviation $\sigma_{r}$ from a square array, s. Fig.1. The full line refers to a system with the same size for all islands, the dashed line to a system with $20 \%$ size dispersion. The line denoted by 'random' refers to $\overline{E_{\text {dip }}}$ of a random island arrangement in the unit cell. For the atomic magnetic moment and the interatomic distance we have choosen values appropriate for Fe: $\mu_{a t}=2.2 \mu_{B}$ and $a_{o}=2.5 \AA$. 